\title{
Pressure Dependence of the Interfacial Structure of Potassium Chloride Films on Iron
}

\author{
Dustin Olson ${ }^{1}$, Hongyu Gao ${ }^{2}$, Chun Tang ${ }^{2}$, Wilfred T. Tysoe ${ }^{1}$ and Ashlie Martini ${ }^{2}$ \\ ${ }^{1}$ Department of Chemistry and Laboratory for Surface Studies, University of Wisconsin- \\ Milwaukee, Milwaukee, WI 53211, USA \\ ${ }^{2}$ School of Engineering, University of California Merced, Merced CA 95343, USA
}

\begin{abstract}
Potassium chloride films on a clean iron surface are used as a model system to explore the interfacial structure of the films and the dependence of that structure on film thickness and pressure. The interfacial structure of one-, two-, three- and four-layer films is measured experimentally using low-energy electron diffraction. Those finding are then complemented by molecular dynamics simulations in which the atomic interaction between the film and substrate is tuned to match film thickness-dependent sublimation activation energy obtained from temperature-programmed desorption measurements. The resultant simulation reliably predicts the structure of thicker films and is then used to study the effect of pressure on the distribution of the lattice constant within and between each layer of the potassium chloride films. Findings indicate that both film thickness and pressure affect the structure within the films as well as the degree of registry between the film and adjacent substrate.
\end{abstract}

Keywords: Boundary lubrication, potassium chloride, molecular dynamics simulations, pressure dependence.

\section{Introduction}

The registry between a film and substrate has a profound influence on interfacial shear. A film that is in registry with the substrate will result in the largest friction force since all the atoms at the interface will simultaneously surmount the sliding potentials. Conversely, a lack of commensurability between the atoms in the contacting interface would lead to a lower shear strength which does not scale linearly with contact area [1-5]. For thin films, commensurability may be dependent on the thickness of the film and, in the case of confined films, pressure. Therefore, understanding the friction properties of an interface requires first understanding the dependence of film structure on thickness and pressure.

$-1-$

(C) 2015. This manuscript version is made available under the Elsevier user license

http://www.elsevier.com/open-access/userlicense/1.0/ 
This complex relationship has been explored using a simple alkali halide as a model boundary lubricating film. While alkali halides are not used as commercial boundary films, their structural simplicity and the availability of a wide range of alkali halides with varying lattice constant, along with their chemical inertness make them attractive candidates for fundamental study [6]. In particular, the shear strength $S$ of $\mathrm{KCl}$ films has been measured experimentally in ultrahigh vacuum (UHV) where it is found that $S$ varies linearly with contact pressure $P$; $S(P)=S_{0}+\alpha P$ where $S_{0}$ and $\alpha$ are constants [7]. Such linear variations in interfacial shear strength as a function of contact pressure are relatively common in tribological systems [8-10]. The specific system explored both in the previous and current study is $\mathrm{KCl}$ on $\mathrm{Fe}(100)$, which is particularly well suited for such studies since precise interaction potentials for computational models are already available for alkali halide films and it is relatively straightforward to prepare well-defined films in ultrahigh vacuum by $\mathrm{KCl}$ evaporation.

This model system has previously been investigated using density-functional theory (DFT) calculations of $\mathrm{KCl}$ films on an $\mathrm{Fe}(100)$ substrate [11]. However, the requirements of using periodic boundary conditions for the calculations necessitated using $\mathrm{KCl}$ films that were in registry with the iron substrate. Since the lattice mismatch between $\mathrm{KCl}$ and the 100 face of iron is significant $(\sim 7 \%)$, the resulting $\mathrm{KCl}$ film is strained. Nevertheless, these calculations revealed a relatively low barrier to sliding the $\mathrm{KCl}$ film against the $\mathrm{Fe}(100)$ face of $\sim 0.7 \mathrm{~kJ} / \mathrm{mol}$ that yielded a calculated shear strength that was in good agreement with experiment. In this case, it was proposed that the pressure-dependent shear strength arose because of the vertical motion of the atoms in the contact as they slid from one stable site to the next over an energy barrier, analogous to the barrier height in the Prandtl-Tomlinson model for sliding friction [12-17]. However, the low value of the sliding potential implies that the $\mathrm{KCl}$ film does not have a strong site preference on the $\mathrm{Fe}(100)$ substrate suggesting that $\mathrm{KCl}$ might not, in fact, be in registry with the Fe substrate and also emphasizes the need for accurate potentials to describe sliding friction.

Although these results show that the previous DFT calculations yield a potential barrier that is in good agreement with the experimentally measured shear strength for $\mathrm{KCl}$ films, there are limitations to the predictions that can be made using this approach given the relatively small simulation size and required periodic boundary conditions which force registry. This suggests the use of molecular dynamics (MD) simulations, which have been extensively used to explore the structural and tribological properties of sliding solid-solid interfaces [18-25]. Such simulations have proven extremely useful in helping to understand the behavior of buried interfaces that, in most cases cannot be interrogated directly. However, MD simulations generally use generic potentials that have been optimized to provide reasonable agreement with the experimental results for a wide range of materials, rather than precisely mimicking the behavior of a specific material. When the interfacial structure is controlled by small energy differences, as is the case in the $\mathrm{KCl}-\mathrm{Fe}$ system, this may result in the prediction of erroneous structures and the resulting properties. Since it is not generally possible to experimentally interrogate the buried sliding interface, the results of such simulations cannot be directly compared with experiment except to compare predicted parameters such as friction force with the experimental value. It is therefore desirable to tune the interaction parameters in the MD simulation to reproduce experimentally measurable film properties measured for the film-surface interface and then use the tuned simulation to provide information about that interface not available via experiment. This is the approach taken in this work. We adjust the interaction potentials between a model alkali halide boundary film on an $\mathrm{Fe}(100)$ substrate by comparison with the results of temperature- 
programmed desorption (TPD) experiments of the desorption behavior of the films. The structure of thin $\mathrm{KCl}$ films is then further characterized experimentally using low-energy electron diffraction (LEED) [26] measurements. Finally, the MD simulations, with interaction potentials fitted to the TPD data, are used to explore the influence of contact pressure on the interfacial structure.

\section{Methods}

1) Experimental Methods

LEED and TPD measurements were carried out in a UHV chamber operating at a base pressure of $\sim 1.3 \times 10^{-8}$ Pa following bakeout. The chamber was pumped by means of a liquid-nitrogentrapped diffusion pump, and the chamber contained a double layer of $\mu$-metal, to minimize extraneous magnetic fields inside the vacuum chamber. The $\mathrm{Fe}(100)$ single crystal (Princeton Scientific, $99.999 \%$ ) purity was initially treated by heating in a hydrogen atmosphere under a pressure of 1 atmosphere at $800 \mathrm{~K}$ for $\sim 420$ hours to reduce the level of bulk carbon in the sample. The sample was then attached to the end of a co-axial sample manipulator, and could be resistively heated to $\sim 900 \mathrm{~K}$ or cooled to $\sim 80 \mathrm{~K}$ by contact with a liquid-nitrogen filled reservoir. The sample temperature was measured by means of a chromel/alumel thermocouple spot welded to the edge of the sample. The sample was then cleaned by repeated argon ion bombardment cycles $\left(4 \mu \mathrm{A} / \mathrm{cm}^{2}\right.$ with a beam energy of $\left.0.5 \mathrm{keV}\right)$ at an elevated sample temperature $(600-700 \mathrm{~K})$ followed by annealing (800-900 K). Auger spectroscopy showed that the iron single crystal was clean after this procedure and exhibited a sharp LEED pattern indicating that the surface was well ordered.

Potassium chloride was evaporated from a small ( $\sim \mathrm{mm}$ internal diameter) alumina tube furnace and the $\mathrm{KCl}$ temperature monitored by means of a chromel/alumel thermocouple placed in the $\mathrm{KCl}$ pellet to ensure a reproducible evaporation rate, which was monitored by means of a watercooled, quartz-crystal microbalance in the UHV chamber. The microbalance was mounted to a z-motion drive to allow it to be moved to the same location as the Fe(100) sample to ensure identical $\mathrm{KCl}$ fluxes during both source calibration and sample dosing. $\mathrm{KCl}$ was deposited with the sample temperature held at $\sim 500 \mathrm{~K}$ to provide a uniform $\mathrm{KCl}$ film [27].

LEED patterns were collected using a four-grid LEED system (Varian) and recorded with a digital camera. Since $\mathrm{KCl}$ is susceptible to electron beam damage where the halide ions are removed via a Knotek-Fiebelman [28, 29] mechanism in which a core hole in the halide ion created by the incident electron beam decays to emit an Auger electron, thereby creating an unstable $\mathrm{Cl}^{+}$ion, all LEED measurements were made using beam energies that were below the $\mathrm{Cl} 1 \mathrm{~s}$ ionization energy.

Temperature-programmed desorption (TPD) data were collected using a heating rate of $5 \mathrm{~K} / \mathrm{s}$ and desorbing species were detected using a (Hiden) quadrupole mass spectrometer whose ionizer was placed in-line-of-sight of the front face of the sample. Both the sample temperature and the ion intensity were collected simultaneously and exported in Excel format. The desorption data were numerically fit to a rate equation of the form $\frac{d \theta}{d t}=-A \exp \left(\frac{E_{a c t}}{R T}\right) \theta^{n}$, where $A$ is a preexponential factor, $E_{a c t}$ is the desorption activation energy, $\Theta$ is the $\mathrm{KCl}$ coverage and $n$ the 
reaction order, using the experimental heating rate. The rate equation was numerically integrated with temperature steps that were sufficiently small $(0.5 \mathrm{~K})$ to yield results that were independent of choice of the temperature step. The parameters were varied to reproduce the experimental desorption maxima and the peak widths and shapes.

\section{2) Molecular Dynamics Simulations}

Two computational models were created as shown in Figure 1. Both models contain an Fe substrate with dimensions of $12.0 \times 12.0 \times 0.6 \mathrm{~nm}^{3}$. A small slab of an $m$-layer $(m=1,2, \ldots, 14)$ $\mathrm{KCl}$ film with in-plane dimensions of $4.72 \times 4.72 \mathrm{~nm}^{2}$ was placed in the center of the Fe. In Model 1, shown in Figure 1(a), the top surface of the $\mathrm{KCl}$ was free. In Model 2, shown in Figure 1(b), a rigid Fe plate, with the same orientation as the bottom Fe surface, was placed on top of the $\mathrm{KCl}$ film. During the simulation, the outermost atomic layer of Fe was held fixed. In both models, periodic boundary conditions were applied in the plane parallel to the $\mathrm{Fe}-\mathrm{KCl}$ interface. Both models were equilibrated by running dynamics for $0.5 \mathrm{~ns}$ until the potential energy of the system reached a constant value. Then, the $\mathrm{KCl}$ lattice was characterized throughout the film by measuring the distance between two adjacent $\mathrm{K}$ ( or $\mathrm{Cl}$ ) atoms in each $\mathrm{KCl}$ layer, and by fitting the distribution of the data to a Gaussian function to obtain the mean lattice constant (for the standard unit cell of the $\mathrm{NaCl}$ (B1) structure) and its standard deviation. Model 2 was used to investigate the effect of pressure with normal loads of $0.014,0.14,1.41$ and $14.1 \mathrm{nN}$ applied to the top plate, corresponding to contact pressures of 0.63, 6.30, 63.0 and 630 MPa. The system was equilibrated for $1 \mathrm{~ns}$ after each load was applied. We partially validated this model by calculating the Poisson's ratio and elastic modulus from the change in the normal and in-plane dimensions of the innermost four layers of the 8-layer $\mathrm{KCl}$ model due to an increase in pressure from 6.3 to $630 \mathrm{MPa}$. The model predicted a Poisson's ratio of 0.194 and an elastic modulus of $44.8 \mathrm{GPa}$, which are reasonably consistent with the bulk properties of $\mathrm{KCl}$ (Poisson ratio = 0.216 ; elastic modulus $=38.2 \mathrm{GPa})$.

The desorption activation energy $\left(E_{a}\right)$ was calculated using Model 1 from the difference in potential energy of the system before and after removing a single $\mathrm{KCl}$ pair. $E_{a}$ was calculated using the equation [30]: $E_{a}=E_{N-1, K C l-F e}+E_{l, K C l}-E_{N, K C l-F e}$, where $E_{N, K C l-F e}$ and $E_{N-1, K C l-F e}$ are the potential energies of the system before and after the removal of $\mathrm{KCl}$, respectively, and $E_{1, \mathrm{KCl}}$ is the energy of one $\mathrm{KCl}$ at equilibrium. The values of $E_{N, \mathrm{KCl}-\mathrm{Fe}}$ and $E_{N-1, \mathrm{KCl}-\mathrm{Fe}}$ were calculated directly from the simulation after energy minimization. However, this approach could not be applied to $E_{l, K C l}$ because the empirical potential was fit to crystalline $\mathrm{KCl}$ and therefore not expected to be able to predict gas-phase energies, which is the state of the $\mathrm{KCl}$ as it is desorbed in the experiment. To address this, we assumed that $E_{a}$ from MD and experiment should be the same for sufficiently large films, i.e. where the Fe interaction has no effect on activation energy, and the value of the calculated $E_{1, K C l}$ was adjusted such that the activation energy of the $2.5 \mathrm{~nm}$ thick film was the same in the experiment and simulation. We initially tested removing $\mathrm{KCl}$ from four different positions on the film, i.e. film center, edge center, and the K- and Cl-terminated corners; see Figure 1(a). The results showed that, for any film thickness, the smallest $E_{a}$ was always calculated from the model where we removed $\mathrm{KCl}$ from one of the two corners. This is consistent with $1 / 2$ order dependence of the activation energy from the TPD experiment (see 
below) which suggested atoms were removed from the edge of the $\mathrm{KCl}$ slab. Therefore, in subsequent calculations, $E_{a}$ was identified as the smaller of the values calculated from the two corner positions.

Multiple empirical potentials were used to describe the atomic interactions for the system. The Born-Mayer-Huggins (BMH) [31] potential was used to model interactions within the $\mathrm{KCl}$, with potential parameters from Ref. [32]. The Embedded-Atom Method (EAM) [33, 34] was used to describe $\mathrm{Fe}$, with potential parameters from Ref. [35]. Interactions between $\mathrm{KCl}$ and $\mathrm{Fe}$ were modeled using the Morse potential: $E=D_{0}\left[e^{-2 \alpha\left(r-r_{0}\right)}-2 e^{-\alpha\left(r-r_{0}\right)}\right]$, where $D_{0}$ is the potential well depth, $r$ is the distance between each atom pair, $r_{0}$ is the equilibrium distance, and $\alpha$ is the exponential parameter that controls the width of the potential well. The potential parameters were tuned by matching the MD-predicted activation energies with the corresponding experimental results. All simulations were conducted using the open-source software LAMMPS [36].

\section{Results and Discussion}

1) Activation Energy

a) Temperature-Programmed Desorption Experiments.

A series of temperature-programmed desorption data for $\mathrm{KCl}$ evaporated onto $\mathrm{Fe}(100)$ at $300 \mathrm{~K}$ collected using a heating rate of $5 \mathrm{~K} / \mathrm{s}$ are displayed in Fig. 2(a) while monitoring $39 \mathrm{amu}$. The desorption profiles are asymmetric indicating a desorption order of unity or less [37] and show a peak temperature that increases as a function of film thickness from $\sim 639 \mathrm{~K}$ for the thinnest films up to $\sim 690 \mathrm{~K}$ for a thick $\mathrm{KCl}$ layer. The inset to Figure 2(a) shows the integrated peak areas for each of the desorption profiles plotted versus the initial film thickness. The linearity of the plot indicates that $\mathrm{KCl}$ adsorbs with a constant sticking probability and that a constant flux is provided by the $\mathrm{KCl}$ dosing source.

The TPD data were fit by numerically integrating the rate equation:

$$
-\frac{d \theta}{d t}=A \theta^{n} e^{-\frac{E_{a c t}(\theta)}{k_{B} T}}
$$

where $A$ is a pre-exponential factor, $\theta$ the coverage, $n$ the reaction order, $E_{a c t}(\theta)$ the coveragedependent desorption activation energy, $k_{B}$ the Boltzmann constant and $T$, the temperature. The fit was carried out over small temperature steps using the measured linear heating rate, $\beta=5 \mathrm{~K} / \mathrm{s}$ to yield:

$$
-\frac{d \theta}{d T}=\frac{A}{\beta} \theta^{n} e^{-\frac{E_{a c t}(\theta)}{k_{B} T}}
$$

The size of the temperature step was decreased until no changes in the fitting parameters were observed to ensure that the numerical integration of Eqn. (2) was accurate. The values of $A, n$ and $E_{a c t}(\theta)$ were systematically adjusted to provide the best fit to each desorption profile by 
minimizing the standard deviation between the theoretical fit and the experimental data. The reaction order $n$ was found to be $0.5 \pm 0.1$ suggesting that desorption takes place on the perimeters of $\mathrm{KCl}$ patches on the surface. The resulting values of the desorption activation energies as a function of film thickness are displayed in Figure 2(b). This initially increases rapidly from a value of $\sim 130 \mathrm{~kJ} / \mathrm{mol}$ for the thinnest films, rising rapidly with increasing film thickness to reach an almost constant value of $\sim 230 \mathrm{~kJ} / \mathrm{mol}$ for films thicker than $\sim 0.8 \mathrm{~nm}$, in reasonable agreement with the heat of sublimation of $\mathrm{KCl}$ [38].

\section{b) $\mathrm{MD}$ and the KCl-Fe Pair Potential.}

The experimentally-measured activation energy was used to tune the Morse potential parameters that describe the interactions between $\mathrm{KCl}$ and $\mathrm{Fe}$ in the $\mathrm{MD}$ simulations. We started with thick films, for which the activation energy was unaffected by the iron substrate and therefore determined only by the $\mathrm{KCl}$ potential. As shown in Figure 3, the activation energy of the simulation and experiment are necessarily the same for the thickest films $(\sim 2.5 \mathrm{~nm})$. As the film thickness decreased, the activation energy decreased and the rate of this decrease was determined in the simulation by the KCl-Fe potential. The initial Morse parameters $\left(D_{0}, \alpha\right.$ and $\left.r_{0}\right)$ were obtained by reproducing the change of energy with distance between $\mathrm{KCl}$ and $\mathrm{Fe}$ slabs from the previous DFT calculations $[11,39,40]$. We then tuned the minimum energy for the K-Fe and $\mathrm{Cl}$-Fe interactions $\left(D_{0, \mathrm{~K}-\mathrm{Fe}}\right.$ and $\left.\mathrm{D}_{0, \mathrm{Cl}-\mathrm{Fe}}\right)$ to match the experimentally measured activation energy for films between 0.3 and $1.2 \mathrm{~nm}$ thick. The other two parameters $\left(\alpha\right.$ and $\left.r_{0}\right)$ were unchanged

from their initial values $\left(\alpha_{K-F e}=9.8 \mathrm{~nm}^{-1}, r_{0, K-F e}=0.421 \mathrm{~nm}, \alpha_{C l-F e}=14.0 \mathrm{~nm}^{-1}\right.$ and $r_{0, C l-F e}=0.270$ $\mathrm{nm})$. We observed that activation energy increased monotonically with increasing $\mathrm{KCl}-\mathrm{Fe}$ interaction strength and that the rate of that increase was faster for thinner films and for stronger interactions. The best fit values of the minimum energy parameter were found to be: $D_{0, K-F e}=$ $0.67 \mathrm{~kJ} / \mathrm{mol}$ and $D_{0, \mathrm{Cl}-\mathrm{Fe}}=8.74 \mathrm{~kJ} / \mathrm{mol}$. Note that we did not model films with thicknesses smaller than the lattice constant of a single layer since those corresponded to experimental measurements of partial films which could not be reproduced reliably in the simulations. Figure 3 shows that the activation energy predicted from MD using the best fit Morse parameters for films at least one layer thick was in good agreement with the values obtained from TPD.

2) Film Structure

a) LEED Patterns.

Low-energy electron diffraction images were obtained for thin $\mathrm{KCl}$ films to avoid sample charging, which was further minimized by keeping the beam current as low as possible. Figure 4(a) shows the LEED pattern for clean Fe(100) collected using a beam energy of $\sim 50 \mathrm{eV}$. This displays a square pattern expected of the $\mathrm{Fe}(100)$ face and is used to calibrate the spacing of $\mathrm{KCl}$ films using the standard lattice constant for Fe of $0.287 \mathrm{~nm}$ [41].

Figure 4(b) shows the corresponding LEED pattern for 1 monolayer (ML) of $\mathrm{KCl}$ deposited onto iron at $\sim 500 \mathrm{~K}$, also collected using the same beam energy of $50 \mathrm{eV}$, showing both the $\mathrm{Fe}(100)$ substrate spots and those due to $\mathrm{KCl}$. Here the substrate spots are circled in red and yield the same spot spacing as in Figure 2(a) confirming that identical electron beam energies were used in both cases. The additional spots due to $\mathrm{KCl}$ are aligned with the $\mathrm{Fe}(100)$ substrate. 
Comparing the spot spacings with those of the underlying $\mathrm{Fe}(100)$ substrate gives the lattice constant for the $\mathrm{KCl}$ monolayer to yield a $\mathrm{K}-\mathrm{K}$ (or $\mathrm{Cl}-\mathrm{Cl}$ ) distance of $0.48 \pm 0.01 \mathrm{~nm}$ (compared with $0.4449 \mathrm{~nm}$ for pure $\mathrm{KCl}$ ) [41]. This suggests that the first layer $\mathrm{KCl}$ is slightly expanded compared to the bulk $\mathrm{KCl}$ lattice.

Figure 4(c-e) display the LEED pattern from two, three and four monolayers of $\mathrm{KCl}$ on $\mathrm{Fe}(100)$, again collected using a beam energy of $50 \mathrm{eV}$, where now the $\mathrm{Fe}(100)$ substrate diffraction spots are obscured. The LEED patterns are slightly distorted since the electron beam is not normal to the surface. However, the spacings between the diffraction spots are identical for all three films indicating that a bulk-like $\mathrm{KCl}$ film is formed when the film thickness is greater than or equal to two monolayers. In addition, the measured spot spacings are consistent with the bulk lattice structure of $\mathrm{KCl}$. This implies that the interaction potential between the $\mathrm{KCl}$ film and the underlying $\mathrm{Fe}(100)$ lattice is small since a two-layer film of $\mathrm{KCl}$ provides sufficient elastic energy to overcome the small lattice distortion (of $\sim 7 \%$ ) found for the first monolayer. Note that even the distorted first-monolayer film is not in registry with the substrate.

\section{b) MD Modeled $\mathrm{KCl}$ with a Free Surface}

In the simulations, Model 1 was used to study the equilibrium structure of $\mathrm{KCl}$ films between 1 and 14 layers thick. Because of the atomic-level detail available in the simulation, we were able to observe the variation of this structure, both between and within the $\mathrm{KCl}$ layers in a given film. Therefore, we characterized the distribution of in-plane lattice constants for each film, using a Gaussian fit to estimate mean values and their standard deviations. Figure 5(a) shows a representative distribution and fit for eight-layer $\mathrm{KCl}$. In this case, the mean lattice constant is comparable to the value predicted for the bulk material using the $\mathrm{BMH}$ potential $(0.625 \mathrm{~nm})$ and the standard deviation is small, indicating that most of the film conforms to the bulk material structure. The mean of the lattice constant for films between 1 and 14 layers thick is shown in Figure 5(b). The mean lattice constants of the thicker model films are comparable to that of bulk $\mathrm{KCl}$, consistent with the LEED pattern for $\mathrm{KCl}$ films of two monolayers and thicker. Note that the mean values reported here were calculated from Gaussian fits to atom positions throughout the film, including the edges of the film where variation from bulk structure is expected. However, recalculation using only the middle $75 \%$ of the films yielded slightly smaller error bars, but similar mean values and the same trends.

However, as the film thickness decreases, so too does the mean lattice constant of the film. For the two-layer film, the mean lattice constant is $0.611 \mathrm{~nm}$, which is between that of bulk $\mathrm{KCl}$ and bulk Fe. This result differs from the LEED pattern which indicated the two-layer film exhibited the bulk $\mathrm{KCl}$ lattice. The mean lattice constant of the model film reflects the relatively large variation of the structure within and between the two layers of $\mathrm{KCl}$. This is reflected by the large error bar for this case in Figure 5(b). It can also be observed from the distribution of the lattice constants in each layer of the model film, which is shown as a contour plot in Figure 6 for a smaller $\mathrm{KCl}$ patch $(2.2 \mathrm{~nm} \times 2.2 \mathrm{~nm})$ to highlight key trends. The contour plot for each given layer was generated by calculating the in-plane mid-point position and the in-plane distance between each pair of adjacent $\mathrm{K}$ ( or $\mathrm{Cl}$ ) atoms. The color on the plots represents the magnitude 
of the distance (lattice) at each mid-point position, with color between calculated values obtained using interpolation.

Figure 6 shows that the first $\mathrm{KCl}$ layer (adjacent to the $\mathrm{Fe}$ ) is predominantly in registry with the $\mathrm{Fe}$, particularly near the center of the film, while the second layer largely reflects the $\mathrm{KCl}$ lattice. It is noted that the escape depth for 50-eV electrons used for the LEED measurements is $\sim 0.4 \mathrm{~nm}$ and thus predominantly probes just the outermost layer of the $\mathrm{KCl}$ film. The difference between the structure of thin films in the experiment and simulation is even more obvious in the singlelayer film. In the simulations, the single layer $\mathrm{KCl}$ mean lattice constant was found to be 0.572 nm, i.e. similar to bulk $\mathrm{Fe}$, indicating that the $\mathrm{KCl}$ is in registry with the Fe substrate. This contrasts with the LEED results which suggest that the single layer $\mathrm{KCl}$ lattice is larger than that of both bulk $\mathrm{Fe}$ and $\mathrm{KCl}$. We were unable to reproduce this observation with any values of the Morse parameters: with strong interactions, the $\mathrm{KCl}$ was in registry with the $\mathrm{Fe}$ as expected for the Morse pair potential; but with weak interactions, the BMH potential that was fitted to bulk $\mathrm{KCl}$ was unable to maintain the single layer structure resulting in a buckling of the film. This issue might be addressed by using a non-pairwise, or location dependent $\mathrm{KCl}-\mathrm{Fe}$ potential, but is out of the scope of this study.

\section{c) Effect of Pressure on Interfacial Structure}

Next we used the simulations to explore the effect of pressure on the structure of $\mathrm{KCl}$ confined between two Fe surfaces (Model 2). We analyzed only films with four layers or more since, as shown in the previous section, thinner model films exhibited structures that differed from the LEED measurements. First we analyzed the variation of structure across the film. The mean inplane lattice in each layer of an eight-layer $\mathrm{KCl} \mathrm{slab}$ as a function of load is shown in Figure 7. At any load, the smallest lattice is observed in layers adjacent to the Fe and the largest in the middle of the film. Comparing the structure of the film at the two loads, we observe that the mean lattice constant of the outermost layers decreases with load while that of the innermost layers increase with load. This suggests that, as load increases, the outermost $\mathrm{KCl}$ layers are forced into registry with the $\mathrm{Fe}$, and that the inner $\mathrm{KCl}$ layers respond to this strain by expanding. The net effect is that there is a slight increase in the overall lattice of the film as the load increases (see dashed lines in Figure 7).

These trends are illustrated in another way in Figure 8, which plots the effect of load on the inplane lattice averaged over the entire eight-layer film, and only the innermost two layers or only the outermost two layers; the mean lattice constant of the free surface film is also shown for reference. Consistent with the trends shown in Figure 7, we observe that the overall mean lattice constant of the confined film is larger than that of the free surface film at any load, and increases slightly with increasing load. This trend is attributable to combined effects of the load-induced increase in the lattice constant of the inner layers and decrease of the lattice constant in the outer layers.

The trends observed for the eight-layer case are also exhibited by the other films. The percent change in the in-plane lattice due to confinement and due to an increase in load by a factor of 1000 for films up to fourteen layers thick is shown in Figure 9. The hollow symbols represent 
the change in the lattice of just the outermost layers, which is negative (smaller lattice) for all films and is relatively constant with varying film thickness. The effect of confinement and load on the overall mean lattice, however, is affected by film thickness. Except for the four-layer film, the change in mean lattice is positive (larger lattice) due to both confinement and load, and these effects increase with film thickness, approaching a constant value above approximately ten layers. Although all of these changes are very small $(<1 \%)$, since the thicker films have more inner layers, the trends are consistent with the suggestion that load is partially accommodated by expansion of the inner layers of a confined film.

\section{Conclusions}

A simple model system consisting of $\mathrm{KCl}$ films on a clean iron surface was used to investigate the effects of thickness and pressure on film structure. The study was performed with both experiments and molecular dynamics simulations. The simulations used existing empirical potentials to describe the $\mathrm{KCl}$ film and $\mathrm{Fe}$ substrate, but the critical interactions between the film and substrate were tuned to match the corresponding experimental system. This was accomplished by performing TPD measurements and fitting that data to a rate equation to yield film thickness-dependent desorption activation energy. The desorption activation energy was then predicted by the simulations, and the minimum energy in the pair potential that was used to describe the $\mathrm{KCl}-\mathrm{Fe}$ interactions was tuned such that the model predictions were consistent with the data obtained from experiment.

LEED measurements $\mathrm{KCl}$ films indicated that two-layer films and thicker films exhibited the structure of bulk $\mathrm{KCl}$ while the single layer film adopted a structure distorted by the adjacent $\mathrm{Fe}$. A model structure of the single layer $\mathrm{KCl}$ film was proposed based on the LEED observations. Unfortunately, simulations were unable to reproduce the structure indicated by the experiments of the thinnest films, instead predicting the first layer of the film would be in registry with the Fe. This is likely a limitation of the pair potential used to model the $\mathrm{KCl}-\mathrm{Fe}$ interaction. However, simulations of thicker films did predict a mean lattice constant consistent with the bulk materials, consistent with the two-layer LEED measurements (and similar results assumed for thicker films).

Simulations of films at least four layers thick were then used to investigate the effect of pressure on film and interface structure. Pressure was found to decrease the lattice constant of the outermost layers of the film while increasing the lattice constant of the film's inner layers. These trends were observed for all films, where the effect of pressure on the inner layer was more significant for thicker films. These results suggest an additional possible origin for the pressuredependence of shear strength. It was previously suggested that the vertical motion associated with sliding from one potential minimum to the next on the surface resulted in additional external work being carried out on the system, thereby yielding a pressure-dependent shear strength. However, the above simulations, combined with the experimental results for the filmvacuum interface, suggest an additional possibility, that of a change in interfacial registry at higher contact pressures. Since the shear strength has been found to increase when the boundary film and the substrate are in registry, the increasing registry between the film and substrate at 
higher pressure can also be expected to result in an increase in shear strength with pressure. Clearly, both effects can occur simultaneously and MD simulations of sliding are currently being carried out to explore these effects as well as the influence of interfacial dynamics on the registry between the film and substrate.

Acknowledgements. We gratefully acknowledge support by the National Science Foundation under grant number CMMI-1265742

\section{References}

[1] M. Hirano, K. Shinjo, Atomistic locking and friction, Physical Review B, 41 (1990) 1183711851.

[2] M.H. Muser, L. Wenning, M.O. Robbins, Simple microscopic theory of Amontons's laws for static friction, Physical Review Letters, 86 (2001) 1295-1298.

[3] M.H. Muser, Structural lubricity: Role of dimension and symmetry, Europhys. Lett., 66 (2004) 97-103.

[4] M.H. Müser, M.O. Robbins, Conditions for static friction between flat crystalline surfaces, Physical Review B, 61 (2000) 2335-2342.

[5] M. Peyrard, S. Aubry, Critical behaviour at the transition by breaking of analyticity in the discrete Frenkel-Kontorova model, Journal of Physics C: Solid State Physics, 16 (1983) 1593.

[6] T. Filleter, W. Paul, R. Bennewitz, Atomic structure and friction of ultrathin films of $\mathrm{KBr}$ on $\mathrm{Cu}(100)$, Physical Review B, 77 (2008) 035430.

[7] F. Gao, O. Furlong, P.V. Kotvis, W.T. Tysoe, Pressure dependence of shear strengths of thin films on metal surfaces measured in ultrahigh vacuum, Tribol. Lett., 31 (2008) 99-106.

[8] B.J. Briscoe, D.C.B. Evans, The Shear Properties of Langmuir-Blodgett Layers, Proceedings of the Royal Society of London. A. Mathematical and Physical Sciences, 380 (1982) 389-407. [9] A. Erdemir, R.A. Erck, J. Robles, Relationship of hertzian contact pressure to friction behavior of self-lubricating boric acid films, Surface and Coatings Technology, 49 (1991) 435438.

[10] M.H. Müser, Lubricants under high local pressure: Liquids act like solids, Materialwissenschaft und Werkstofftechnik, 35 (2004) 603-609.

[11] M. Garvey, O.J. Furlong, M. Weinert, W.T. Tysoe, Shear properties of potassium chloride films on iron obtained using density functional theory, J. Phys.-Condes. Matter, 23 (2011) 265003.

[12] L. Prandtl, Ein Gedankenmodell zur kinetischen Theorie der festen Körper., Z. Angew. Math. Mech., 8 (1928) 85.

[13] G.A. Tomlinson, A Molecular Theory of Friction, Phil. Mag., 7 (1929) 905.

[14] E. Gnecco, R. Bennewitz, A. Socoliuc, E. Meyer, Friction and wear on the atomic scale, Wear, 254 (2003) 859-862.

[15] C. Fusco, A. Fasolino, Velocity dependence of atomic-scale friction: A comparative study of the one- and two-dimensional Tomlinson model, Physical Review B, 71 (2005) 045413. [16] O.J. Furlong, S.J. Manzi, V.D. Pereyra, V. Bustos, W.T. Tysoe, Monte Carlo Simulations for Tomlinson Sliding Models for Non-Sinusoidal Periodic Potentials, Tribol. Lett., 39 (2010) 177-180. 
[17] M. Müser, Velocity dependence of kinetic friction in the Prandtl-Tomlinson model, Physical Review B, 84 (2011) 125419.

[18] J.A. Harrison, D.W. Brenner, Simulated Tribochemistry - An Atomic Scale View of the Wear of Diamond, Journal of the American Chemical Society, 116 (1994) 10399-10402.

[19] G.T. Gao, P.T. Mikulski, J.A. Harrison, Molecular-scale tribology of amorphous carbon coatings: Effects of film thickness, adhesion, and long-range interactions, Journal of the American Chemical Society, 124 (2002) 7202-7209.

[20] W.B. Donald, A.S. Olga, A.H. Judith, J.S. Steven, N. Boris, B.S. Susan, A secondgeneration reactive empirical bond order (REBO) potential energy expression for hydrocarbons, Journal of Physics: Condensed Matter, 14 (2002) 783.

[21] J.A. Harrison, G. Gao, J.D. Schall, M.T. Knippenberg, P.T. Mikulski, Friction between solids, Philosophical Transactions of the Royal Society A: Mathematical, Physical and Engineering Sciences, 366 (2008) 1469-1495.

[22] Y. Dong, Q. Li, J. Wu, A. Martini, Friction, slip and structural inhomogeneity of the buried interface, Model. Simul. Mater. Sci. Eng., 19 (2011).

[23] Y. Dong, Q. Li, A. Martini, Molecular dynamics simulation of atomic friction: A review and guide, Journal of Vacuum Science \&amp; Technology A, 31 (2013) 030801.

[24] S.B. Sinnott, S.-J. Heo, D.W. Brenner, J.A. Harrison, Computer Simulations of NanometerScale Indentation and Friction, Nanotribology and Nanomechanics, Springer Berlin Heidelberg2008, pp. 655-740.

[25] I. Szlufarska, M. Chandross, R.W. Carpick, Recent advances in single-asperity nanotribology, Journal of Physics D: Applied Physics, 41 (2008) 123001.

[26] M.A. Van Hove, S.Y. Tong, Surface crystallography by LEED : theory, computation, and structural results, Springer-Verlag, Berlin; New York, 1979.

[27] F. Gao, G. Wu, D. Stacchiola, M. Kaltchev, P.V. Kotvis, W.T. Tysoe, The tribological properties of monolayer $\mathrm{KCl}$ films on iron in ultrahigh vacuum: Modeling the extreme-pressure lubricating interface, Tribol. Lett., 14 (2003) 99-104.

[28] M.L. Knotek, P.J. Feibelman, Ion Desorption by Core-Hole Auger Decay, Physical Review Letters, 40 (1978) 964-967.

[29] P.J. Feibelman, M.L. Knotek, Reinterpretation of electron-stimulated desorption data from chemisorption systems, Physical Review B, 18 (1978) 6531-6539.

[30] H. Zhang, J.R. Rustad, J.F. Banfield, Interaction between Water Molecules and Zinc Sulfide Nanoparticles Studied by Temperature-Programmed Desorption and Molecular Dynamics Simulations, The Journal of Physical Chemistry A, 111 (2007) 5008-5014.

[31] M.J.L. Sangster, M. Dixon, Interionic potentials in alkali halides and their use in simulations of the molten salts, Advances in Physics, 25 (1976) 247-342.

[32] M.C.C. Ribeiro, Chemla Effect in Molten $\mathrm{LiCl} / \mathrm{KCl}$ and $\mathrm{LiF} / \mathrm{KF}$ Mixtures, The Journal of Physical Chemistry B, 107 (2003) 4392-4402.

[33] M.S. Daw, M.I. Baskes, Embedded-atom method: Derivation and application to impurities, surfaces, and other defects in metals, Physical Review B, 29 (1984) 6443-6453.

[34] M.S. Daw, M.I. Baskes, Semiempirical, Quantum Mechanical Calculation of Hydrogen Embrittlement in Metals, Physical Review Letters, 50 (1983) 1285-1288.

[35] M.I. Mendelev, S. Han, D.J. Srolovitz, G.J. Ackland, D.Y. Sun, M. Asta, Development of new interatomic potentials appropriate for crystalline and liquid iron, Philosophical Magazine, 83 (2003) 3977-3994. 
[36] S. Plimpton, Fast Parallel Algorithms for Short-Range Molecular Dynamics, Journal of Computational Physics, 117 (1995) 1-19.

[37] P.A. Redhead, Thermal desorption of gases, Vacuum, 12 (1962) 203-211.

[38] M.W. Chase, S. National Institute of, Technology, NIST-JANAF thermochemical tables, American Chemical Society ; American Institute of Physics for the National Institute of Standards and Technology, [Washington, D.C.]; Woodbury, N.Y., 1998.

[39] M. Garvey, M. Weinert, W.T. Tysoe, On the Pressure Dependence of Shear Strengths in Sliding, Boundary-Layer Friction, Tribol. Lett., 44 (2011) 67-73.

[40] M. Garvey, M. Weinert, W.T. Tysoe, On the film thickness dependence of shear strengths in sliding, boundary-layer friction, Wear, 274-275 (2012) 281-285.

[41] R.W.G. Wyckoff, Crystal structures, Interscience Publishers, New York, 1963. 


\section{Figure Captions}

Figure 1: Snapshots of the two MD models. (a) In Model 1, the top surface of the $\mathrm{KCl}$ is free. White dashed circles identify representative positions (i.e. film center, edge center, $\mathrm{K}$ - and $\mathrm{Cl}$ terminated corners) from which $\mathrm{KCl}$ was removed to calculate activation energy. (b) $\mathrm{The} \mathrm{KCl}$ film is confined between two Fe plates in Model 2 with varying normal loads applied to the upper plate.

Figure 2: (a): Temperature-programmed desorption (TPD) data collected by monitoring 39 am for $\mathrm{KCl}$ adsorbed on a $\mathrm{Fe}(100)$ surface at $300 \mathrm{~K}$ collected using a heating rate of $5 \mathrm{~K} / \mathrm{s}$ and a function of $\mathrm{KCl}$ films thickness. The inset shows a plot of the integrated peak area as a function of film thickness. (b) Plot of desorption activation energy as a function of films thickness obtained by fitting the desorption profiles shown in Figure 2(a).

Figure 3: MD-predicted and experimentally-measured activation energies as functions of film thickness.

Figure 4: LEED patterns collected using a beam energy of $50 \mathrm{eV}$ for (a): a clean Fe(100) surface, (b) $1 \mathrm{ML}$ of $\mathrm{KCl}$ on $\mathrm{Fe}(100)$. The spots due to the $\mathrm{Fe}(100)$ substrate are circled in red, while the additional features are due to $\mathrm{KCl}$. (c) $2 \mathrm{ML}$ of $\mathrm{KCl}$ of $\mathrm{Fe}(100)$ (d) $3 \mathrm{ML}$ of $\mathrm{KCl}$ on $\mathrm{Fe}(100)$ and (e) $4 \mathrm{ML}$ of $\mathrm{KCl}$ on $\mathrm{Fe}(100)$. The substrate spots are no longer visible in the last three diffraction patterns and the spots are due only to the $\mathrm{KCl}$ film.

Figure 5: a) Distribution of in-plane lattice constants within an eight-layer $\mathrm{KCl}$ film, fit to a Gaussian function to enable calculation of the mean and standard deviation. (b) Mean in-plane lattice constant for $\mathrm{KCl}$ films of varying thickness.

Figure 6: Contour plots of the in-plane lattice in each of the two layers of two-layer $\mathrm{KCl}$ illustrating the variation of the structure of the film, both within and between the layers.

Figure 7: Structure of the eight-layer confined $\mathrm{KCl}$ film at two different normal loads. Points represent the mean in-plane lattice constant in each layer of the film where layers 1 and 8 are adjacent to the $\mathrm{Fe}$, and dashed lines represent the overall mean in-plane lattice of the confined film subject to load and with one free surface.

Figure 8: Variation of the mean in-plane lattice constant of the $\mathrm{KCl}$ films due to load. The mean in-plane lattice constant is reported for the entire film and for the innermost and outermost layers of that film. The free surface film in-plane lattice constant is shown for reference.

Figure 9: Change in the mean in-plane lattice constant of a film (solid symbols) and outermost layers (hollow symbols) of that film due to confinement (blue triangles) and a 100 fold increase in load (red circles). 


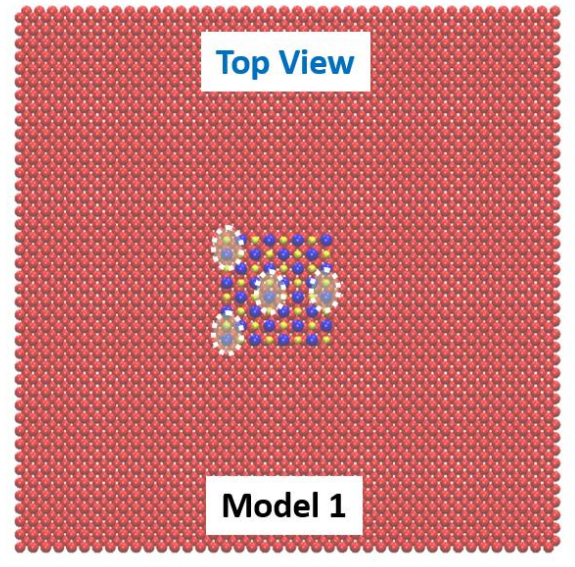

(a)

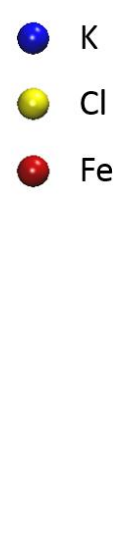

Model 2

\section{Olson et al, Figure 1.}

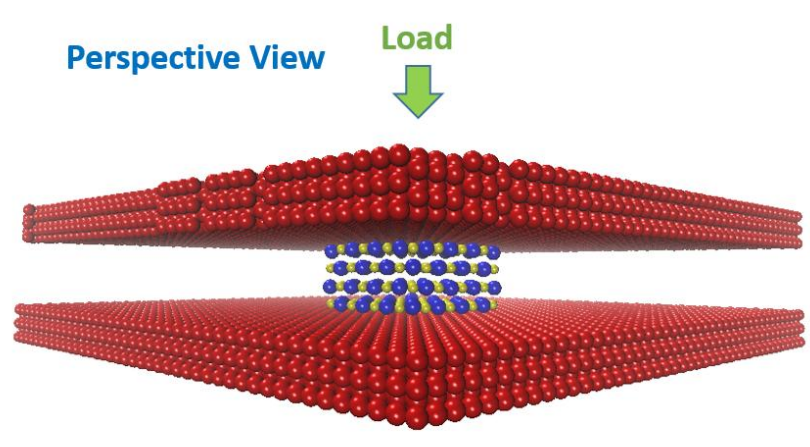

(b) 
(a)

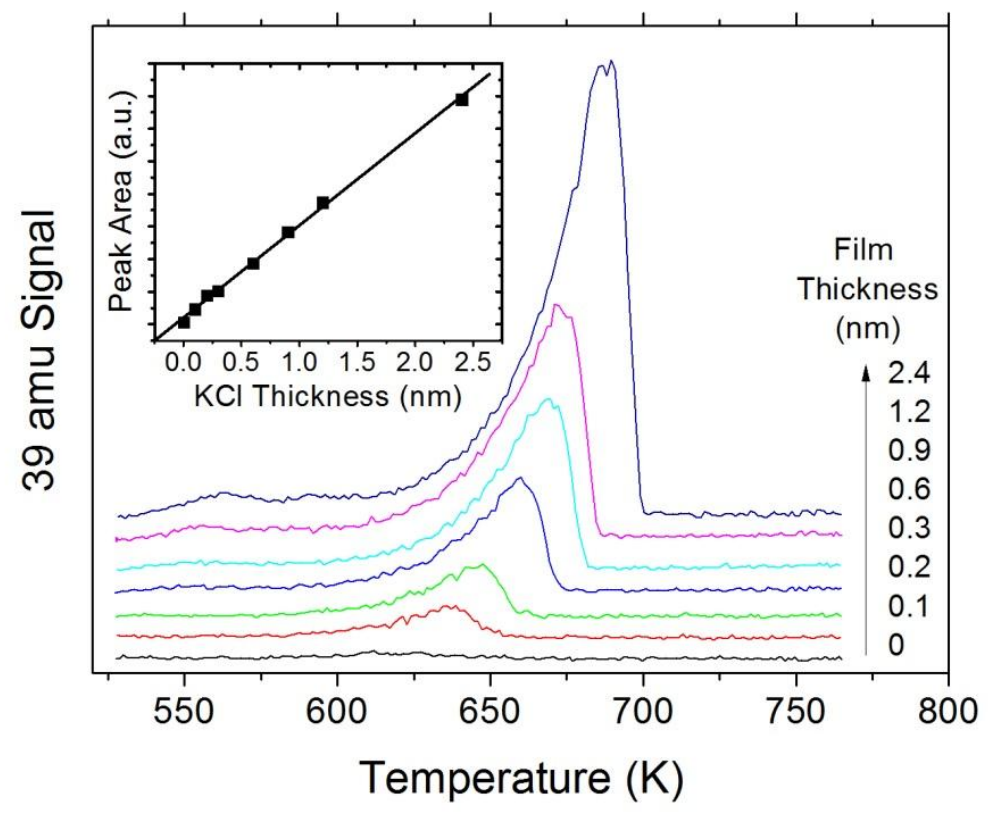

(b)

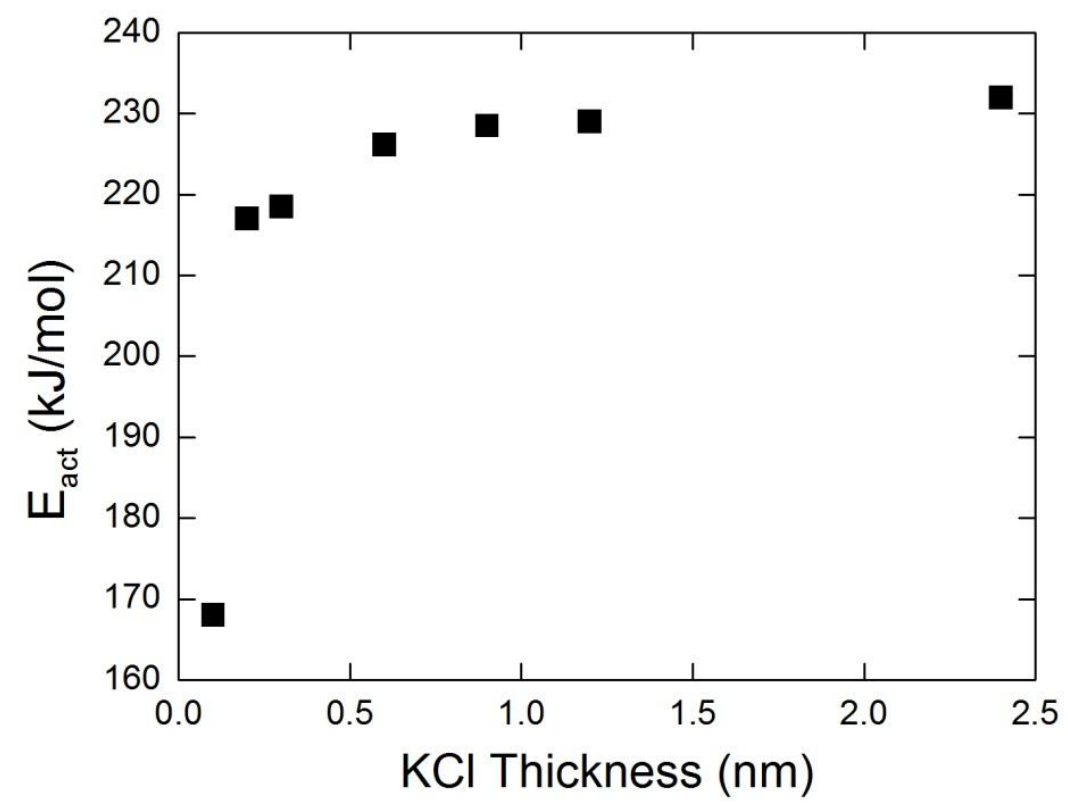

Olson et al, Figure 2. 


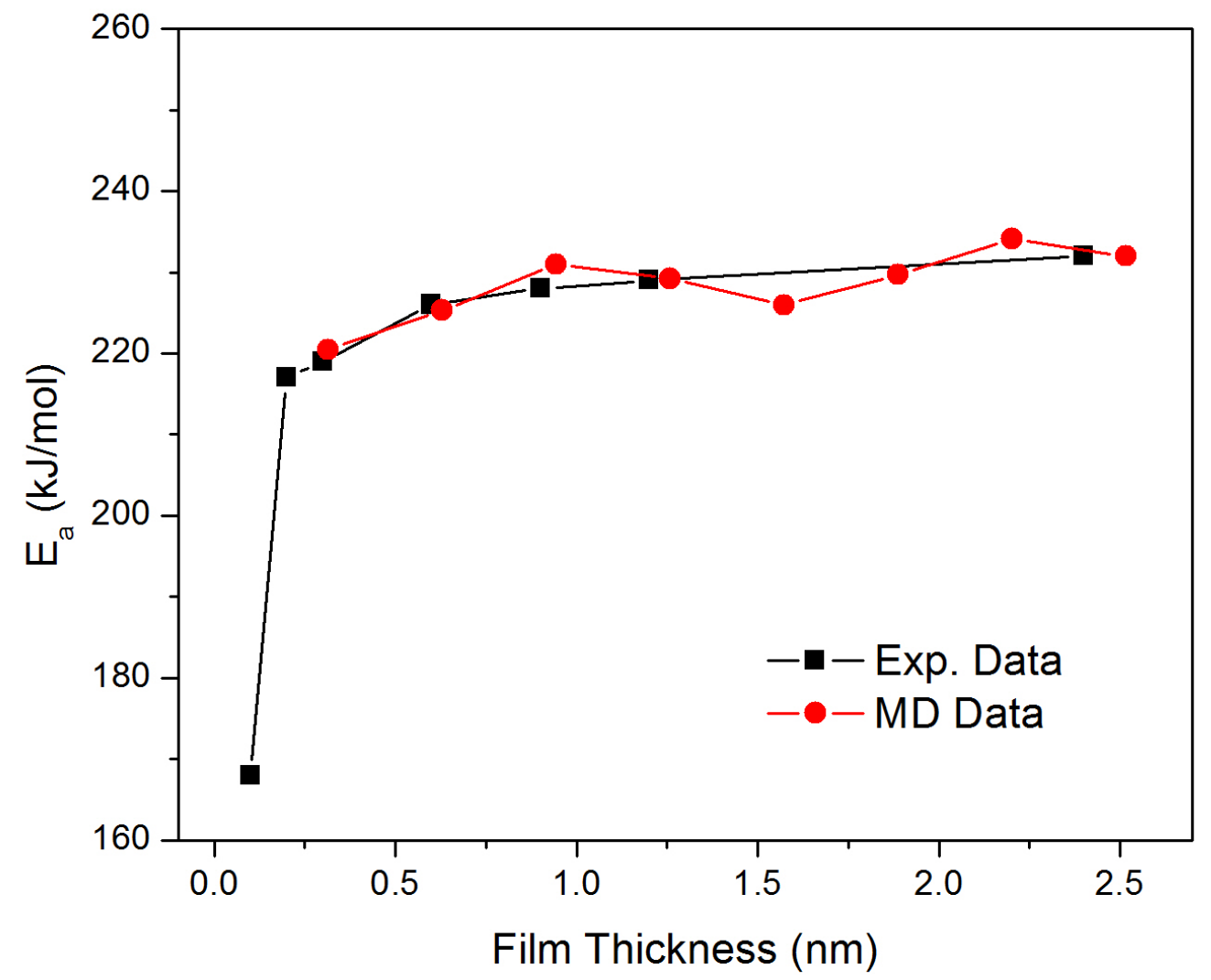

Olson et al, Figure 3. 

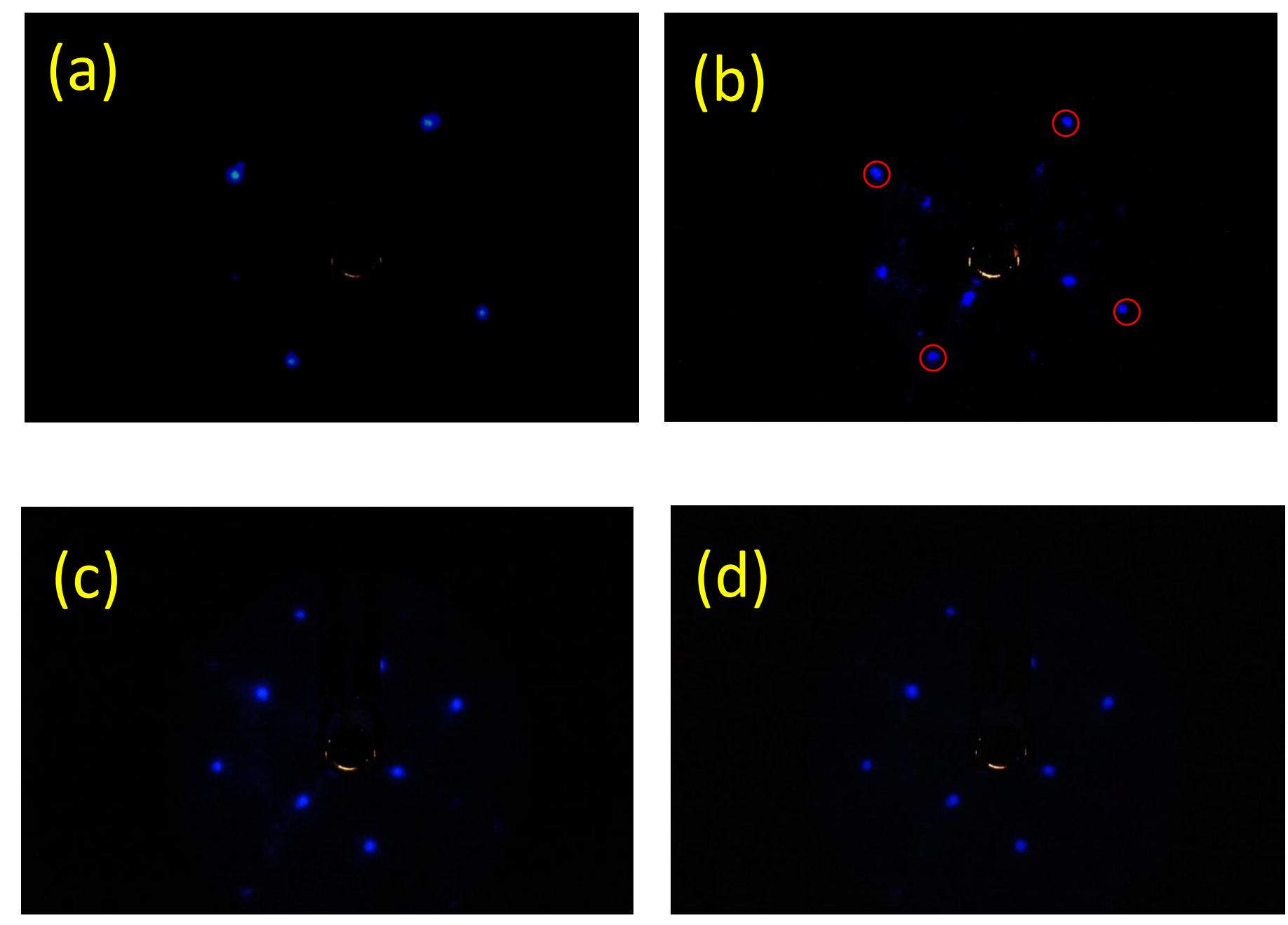

\section{(d)}

(e)

Olson et al, Figure 4. 


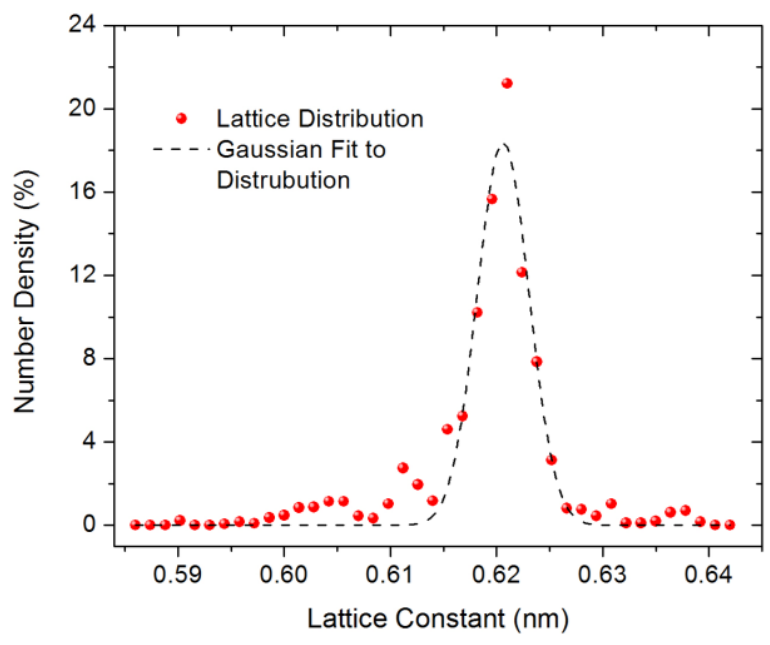

(a)

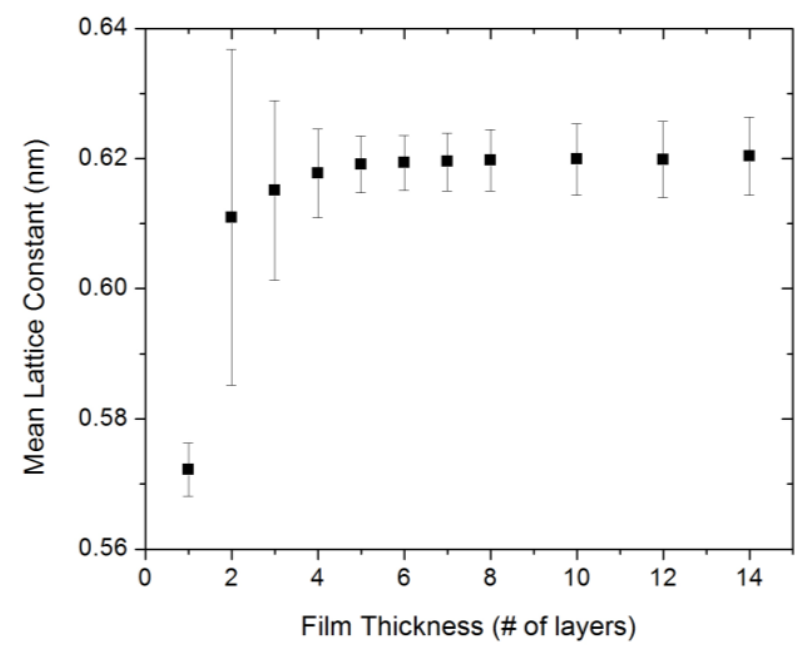

(b)

\section{Olson et al, Figure 5.}




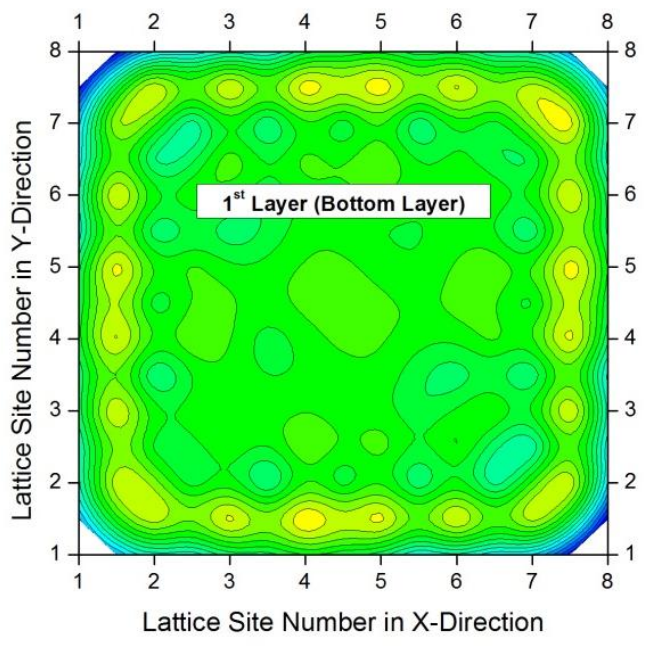

(a)

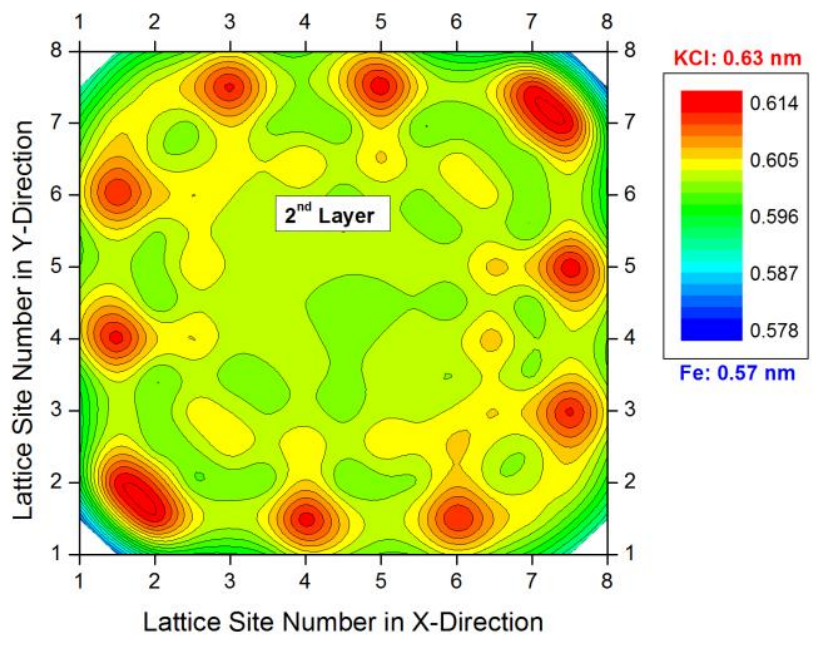

(b)

\section{Olson et al, Figure 6.}




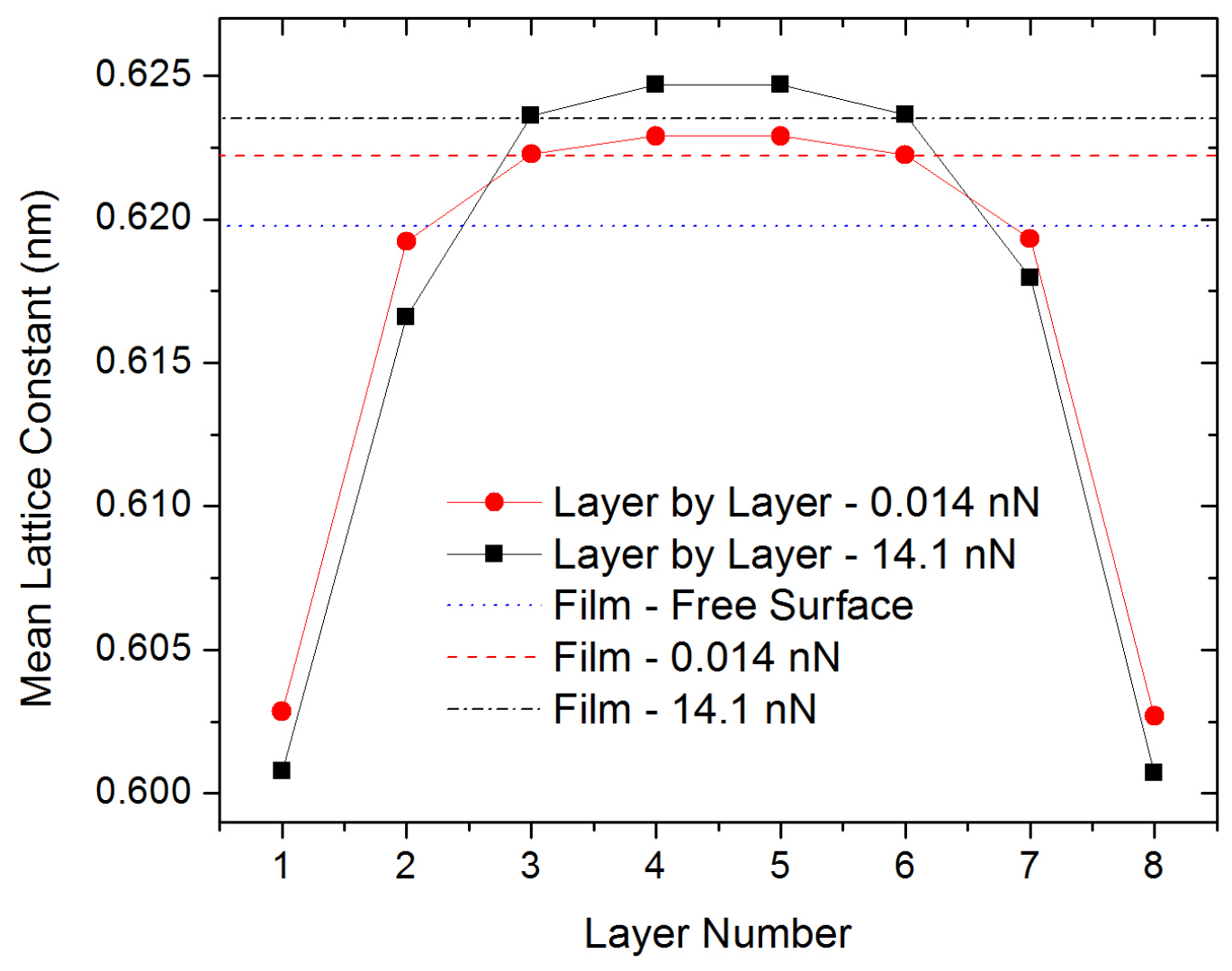

Olson et al, Figure 7. 


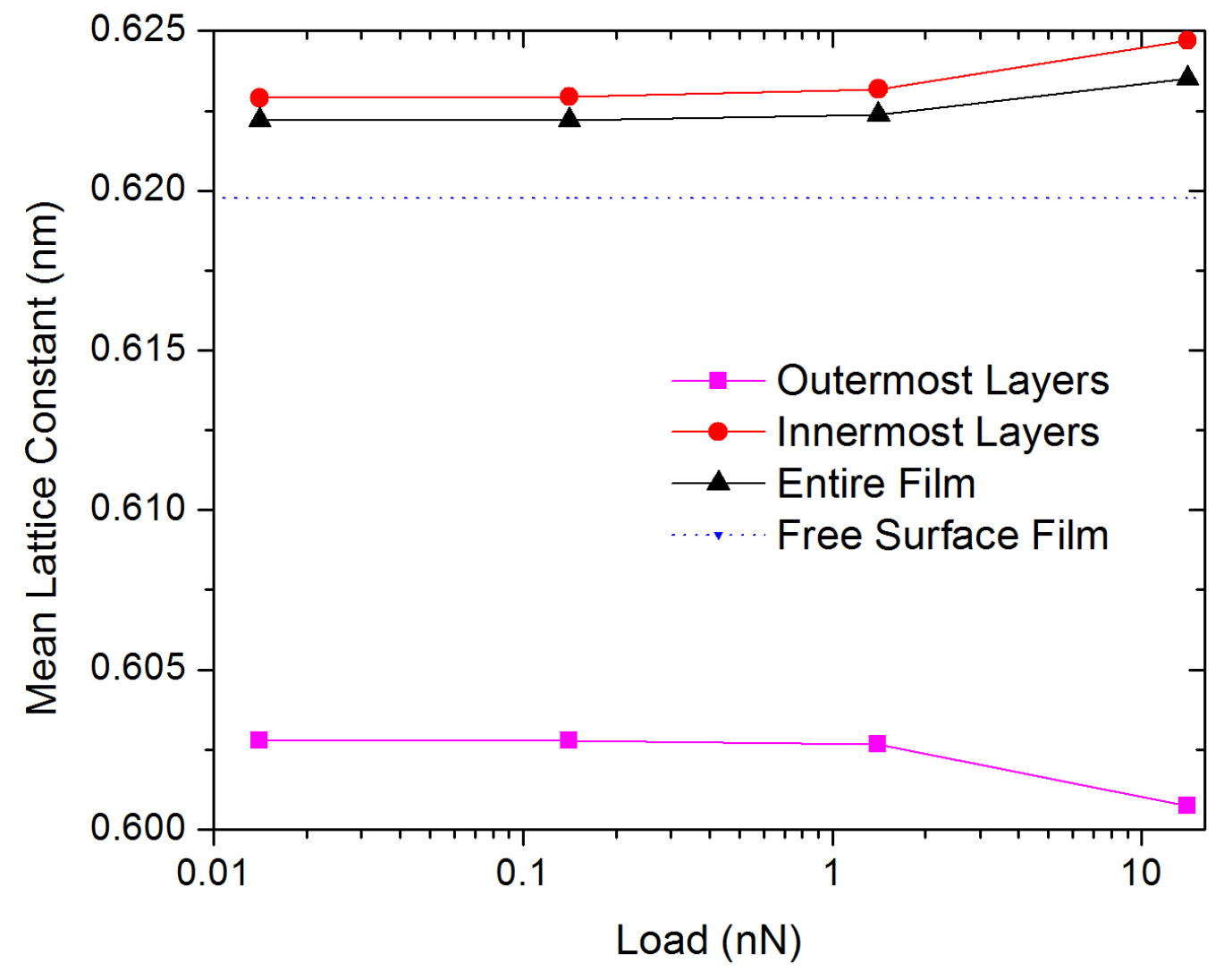

Olson et al, Figure 8. 


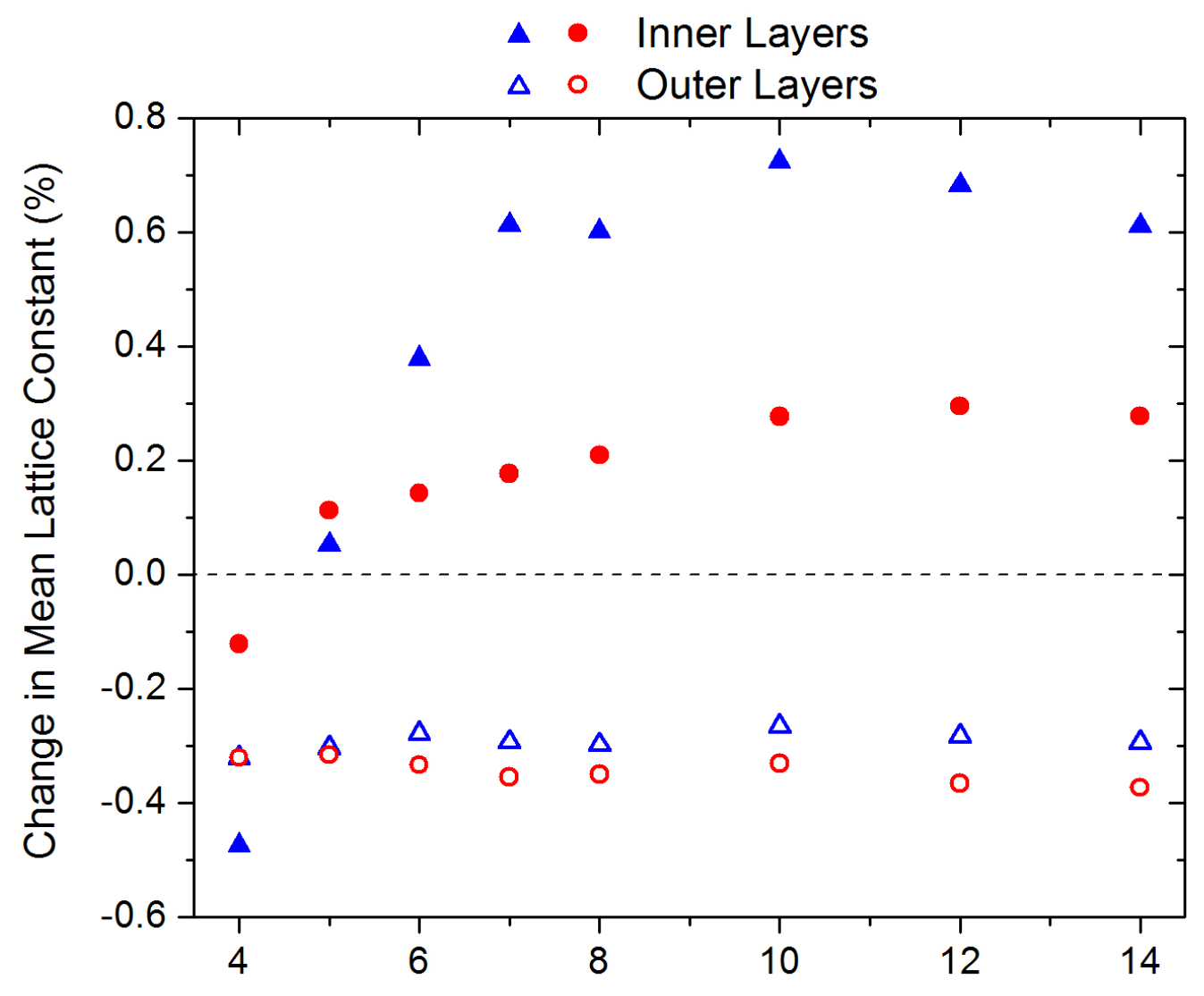

Film Thickness (\# of layers)

\section{Olson et al, Figure 9.}

